\title{
Viscoelastic modelling of regular cross-ply polymer-matrix laminates
}

\author{
M. Klasztorny, T. Niezgoda \& R. Gieleta \\ Department of Mechanics \& Applied Computer Science, \\ Faculty of Mechanical Engineering, Military University of Technology, \\ Warsaw, Poland
}

\begin{abstract}
Recent rheological experiments performed on cross-ply laminates have pointed out that polymer-matrix laminates may exhibit valuable creep shear strains. So far, a problem of viscoelastic modelling has been developed only for UD xFRP composites, i.e. thermosets reinforced with long fibres aligned unidirectionally. In the modelling, the most advanced rheological model of thermosets, denoted with the symbol $\mathrm{HWKK} / \mathrm{H}$, has been employed. This model is capable of modelling short-, moderate- and long-term rheological processes. In this study, the analytical approach developed for UD xFRP composites has been extended on a regular cross-ply polymer-matrix laminate, denoted with the symbol CP $\mathrm{xFRP}$, of the $[0 / 90]_{n \mathrm{~S}}, n \geq 4$ plies' configuration. The plies, made of a specified woven fabric, are repeatable with respect to their thickness and microstructure. On the micromechanics level, each ply is modelled as a linearly viscoelastic monotropic continuum with the monotropy axis coinciding the direction of fibres' alignment. A regular CP xFRP laminate is modelled as a homogeneous orthotropic continuum. Standard and inverse viscoelasticity equations of an orthotropic solid body modelling a homogenized CP xFRP laminate are formulated and analytic algorithms for determination of elasticity and viscoelasticity constants are developed. The calculation algorithms have been computerized and used to calculate the viscoelasticity coefficients of the specified CP CFRP laminate.
\end{abstract}

Keywords: cross-ply polymer-matrix laminates, linear viscoelasticity, standard constitutive equations, inverse constitutive equations, long-term creep coefficients, long-term relaxation coefficients, elastic-viscoelastic analogy principle. 


\section{Introduction}

Recent rheological experiments performed on cross-ply laminates have pointed out that polymer-matrix laminates may exhibit valuable creep shear strains [1]. So far, a problem of viscoelastic modelling has been solved only for UD xFRP composites, i.e. thermosets reinforced with long fibres aligned unidirectionally $[2,3]$. Viscoelastic modelling presented in [2, 3] employs the most advanced rheological model of thermosets, denoted with the symbol HWKK/H, capable of modelling short-, moderate- and long-term rheological processes.

In this study, the analytical approach presented in $[2,3]$ will be developed on a regular cross-ply laminate, denoted with the symbol CP xFRP, of the plies' configuration $[0 / 90]_{n \mathrm{~S}}, n \geq 4$. The plies, made of a specified woven fabric, are repeatable with respect to their thickness and microstructure. This type of laminates is widely used in engineering practice [4].

\section{Assumptions, coordinate systems and sets of elasticity constants}

A regular CP xFRP laminate is manufactured as a stack of plies of the $[0 / 90]_{n \mathrm{~S}}$, $n \geq 4$ configuration. Each ply is a UD xFRP composite. On the micromechanics level, a UD xFRP composite is modelled as a linearly viscoelastic monotropic continuum with the monotropy axis coinciding the direction of fibres' alignment [2]. The following assumptions are adopted:

- each ply is a two-phase material,

- there are considered quasi-static isothermal processes in the normal conditions, i.e. the processes belonging to the transition regime under the glass transition temperature,

- both constituents, i.e. a matrix and a fibre, are homogeneous,

- stresses are restricted to the levels protecting linear behaviour of the constituents,

- a matrix is a chemically hardening plastic made of a crosslinked polymer, modelled as a viscoelastic isotropic material, described by the $\mathrm{HWKK} / \mathrm{H}$ rheological model [5],

- a fibre is modelled as an elastic monotropic material (isotropic, in particular),

- fibres have identical solid circular cross section; they are rectilinear and embedded uniformly in the matrix, in a hexagonal scheme,

- the matrix-fibre interface is a cylindrical surface,

- preparation of the fibres protects perfect bonding of the fibres to the matrix,

- residual stresses resulting from the manufacturing process are neglected,

- the Boltzmann superposition principle is satisfied.

Each ply is described in the $x_{1} x_{2} x_{3}$ - Cartesian coordinate system with $x_{1}-$ a monotropy axis, and $x_{2} x_{3}-$ a transverse isotropy plane. The constituents are characterized by the following elasticity constants: $E, v$ (a Young's modulus 
and a Poisson's ratio of an isotropic matrix), $\bar{E}_{1}, \bar{E}_{2}, \bar{v}_{32}, \bar{v}_{21}, \bar{G}_{12}$ (longitudinal and transverse Young's moduli, Poisson's ratios in respective planes and a shear modulus in the monotropy plane of a monotropic fibre). The composite is also described by the real fibre volume fraction $f$.

A monotropic continuum modelling the homogenized ply is described by five independent effective elasticity constants (EECs), i.e.: $E_{1}, E_{2}, v_{32}, v_{21}, G_{12}$ (effective longitudinal and transverse Young's moduli, effective Poisson's ratios in respective planes, an effective shear modulus in the monotropy plane). These constants are derived with high accuracy, in terms of elasticity constants of the constituents and of the fibre volume fraction, by the exact stiffness theory summarized in [6].

On the mesomechanics level, a regular CP xFRP laminate can be modelled as a homogeneous orthotropic continuum, [7], described in the $x y z$ - Cartesian coordinate system. Axes $x, y$ coincide the lamination directions of respective groups of plies, whereas the $z$ axis is perpendicular to the $x y$ midplane.

For shortening, the symbols LEC and LVC are introduced, respectively denoting constitutive equations of linear elasticity and viscoelasticity.

\section{Standard and inverse LEC equations of CP XFRP}

Standard LEC equations of an orthotropic solid body modelling the homogenized regular CP xFRP laminate have the following form $[4,6]$

$$
\varepsilon=\mathbf{S} \boldsymbol{\sigma},
$$

where

$$
\boldsymbol{\sigma}=\operatorname{col}\left(\sigma_{x}, \sigma_{y}, \sigma_{z}, \sigma_{y z}, \sigma_{x z}, \sigma_{x y}\right), \boldsymbol{\varepsilon}=\operatorname{col}\left(\varepsilon_{x}, \varepsilon_{y}, \varepsilon_{z}, \gamma_{y z}, \gamma_{x z}, \gamma_{x y}\right)
$$

stress and strain vectors in the $x y z$ - system, with classic components: $\sigma_{x}, \sigma_{y}, \sigma_{z}-$ normal stresses, $\sigma_{y z}, \sigma_{x z}, \sigma_{x y}-$ shear stresses, $\varepsilon_{x}, \varepsilon_{y}, \varepsilon_{z}-$ directional strains, $\gamma_{y z}, \gamma_{x z}, \gamma_{x y} \quad-$ shear strains. The elastic compliance matrix $\mathbf{S}$ contains the following well-known elasticity compliances

$$
\begin{gathered}
S_{11}^{\mathrm{cp}}=\frac{1}{E_{x}}, S_{22}^{\mathrm{cp}}=\frac{1}{E_{y}}, S_{33}^{\mathrm{cp}}=\frac{1}{E_{z}}, S_{12}^{\mathrm{cp}}=-\frac{v_{y x}}{E_{x}}, S_{13}^{\mathrm{cp}}=-\frac{v_{z x}}{E_{x}}, S_{23}^{\mathrm{cp}}=-\frac{v_{z y}}{E_{y}}, \\
S_{44}^{\mathrm{cp}}=\frac{1}{G_{y z}}, S_{55}^{\mathrm{cp}}=\frac{1}{G_{x z}}, S_{66}^{\mathrm{cp}}=\frac{1}{G_{x y}},
\end{gathered}
$$

expressed in terms of the EECs of the homogenised laminate, i.e. $E_{x}, E_{y}, E_{z}$ - Young's moduli in the $x, y, z$ directions, $v_{z y}, v_{z x}, v_{y x}-$ Poisson's ratios in respective planes, $G_{y z}, G_{x z}, G_{x y}$ - shear moduli in respective planes. For a regular CP xFRP laminate only six EECs have different values, namely $E_{x}, E_{z}, v_{z x}, v_{y x}, G_{x z}, G_{x y}$. The remaining constants equal $E_{y}=E_{x}, v_{z y}=v_{z x}, G_{y z}=G_{x z}$. 
Based on eqns. (1), (2), inverse LEC equations of an orthotropic solid body have the following matrix form [4]

$$
\boldsymbol{\sigma}=\mathbf{C} \varepsilon
$$

where the elastic stiffness matrix $\mathbf{C}=\mathbf{S}^{-1}$ contains six independent elasticity moduli calculated from the formulae suitable in further considerations, i.e.

$$
\begin{gathered}
C_{11}^{\mathrm{cp}}=\left[S_{11}^{\mathrm{cp}} S_{33}^{\mathrm{cp}}-\left(S_{13}^{\mathrm{cp}}\right)^{2}\right] / \Delta, C_{33}^{\mathrm{cp}}=\left[\left(S_{11}^{\mathrm{cp}}\right)^{2}-\left(S_{12}^{\mathrm{cp}}\right)^{2}\right] / \Delta, C_{55}^{\mathrm{cp}}=1 / S_{55}^{\mathrm{cp}}, \\
C_{12}^{\mathrm{cp}}=\left[\left(S_{13}^{\mathrm{cp}}\right)^{2}-S_{12}^{\mathrm{cp}} S_{33}^{\mathrm{cp}}\right] / \Delta, C_{13}^{\mathrm{cp}}=\left[S_{13}^{\mathrm{cp}}\left(S_{12}^{\mathrm{cp}}-S_{11}^{\mathrm{cp}}\right)\right] / \Delta, C_{66}^{\mathrm{cp}}=1 / S_{66}^{\mathrm{cp}}, \\
\Delta=S_{33}^{\mathrm{cp}}\left[\left(S_{11}^{\mathrm{cp}}\right)^{2}-\left(S_{12}^{\mathrm{cp}}\right)^{2}\right]+2\left(S_{13}^{\mathrm{cp}}\right)^{2}\left(S_{12}^{\mathrm{cp}}-S_{11}^{\mathrm{cp}}\right) .
\end{gathered}
$$

\section{Formulae describing EECs of a regular CP xFRP laminate}

The exact stiffness theory of a regular CP xFRP laminate is formulated in [7]. This theory gives the following final set of analytical formulae predicting EECs of this type of laminates:

$$
\begin{gathered}
E_{x}=E_{y}=\frac{1}{2} a\left(1-b^{2}\right), \quad E_{z}=\frac{a(1+b)}{a(1+b) d+c^{2}}, \\
v_{z x}=v_{z y}=\frac{1}{2} c(1-b), \quad v_{y x}=b, G_{x z}=G_{y z}=\frac{2 G_{12} G_{23}}{G_{12}+G_{23}}, \quad G_{x y}=G_{12},
\end{gathered}
$$

where

$$
\begin{gathered}
a=\frac{E_{1}+E_{2}}{1-v_{21} v_{12}}, \quad b=\frac{E_{1} v_{12}+E_{2} v_{21}}{E_{1}+E_{2}}, v_{12}=\frac{E_{2}}{E_{1}} v_{21}, \quad G_{23}=\frac{E_{2}}{2\left(1+v_{32}\right)}, \\
c=\frac{v_{21}\left(1+v_{12}+v_{32}\right)+v_{32}}{1-v_{21} v_{12}}, \quad d=\frac{1-2 v_{21} v_{12}\left(1+v_{32}\right)-v_{32}^{2}}{E_{2}\left(1-v_{21} v_{12}\right)} .
\end{gathered}
$$

\section{Calculation of the exact complex compliances of CP xFRP}

Complex compliances related to steady-state harmonic processes play a principal role in viscoelastic modelling of xFRP laminates [2, 3]. The exact complex compliances of a homogenized regular CP xFRP laminate can be calculated analytically using the elastic-viscoelastic analogy principle. Calculating the exact complex compliances of a CP xFRP laminate will employ the exact complex compliances of a UD xFRP composite obtained in [2].

Five independent elastic compliances of a UD xFRP composite have been expressed in terms of the elastic shear compliance of a viscoelastic isotropic polymer matrix, $S_{b}=1 / 2 G$ with the shear modulus $G=E /[2(1+v)]$, i.e. 
$S_{i j}\left(S_{b}\right), \quad i j=11,22,12,23,55$ The elastic-viscoelastic analogy principle gives the exact complex compliances $S_{i j}^{*}(p)=S_{i j}^{\prime}(p)+\mathrm{i} S_{i j}^{\prime \prime}(p)=S_{i j}\left[S_{b}^{*}(p)\right]$, $i j=11,22,12,23,55$. Symbol $p$ denotes circular frequency, $i=\sqrt{-1}$, whereas $S_{b}^{*}(p)$ is a complex shear compliance of the matrix, determined in [2] for the $\mathrm{HWKK} / \mathrm{H}$ rheological model. Taking into consideration eqns (2), (5), (6), six independent elastic compliances of a regular CP xFRP laminate can be expressed in terms of the elastic compliances $S_{i j}\left(S_{b}\right), i j=11,22,12,23,55$, in the form

$$
\begin{gathered}
S_{11}^{\mathrm{cp}}=\frac{1}{E_{x}}=\frac{2}{a\left(1-b^{2}\right)}, \quad S_{33}^{\mathrm{cp}}=\frac{1}{E_{z}}=d+\frac{c^{2}}{a(1+b)}, S_{12}^{\mathrm{cp}}=-\frac{v_{y x}}{E_{x}}=-b S_{11}^{\mathrm{cp}}, \\
S_{13}^{\mathrm{cp}}=-\frac{v_{z x}}{E_{x}}=\frac{1}{2}(b-1) c S_{11}^{\mathrm{cp}}, \quad S_{55}^{\mathrm{cp}}=\frac{1}{G_{x z}}=S_{22}-S_{23}+\frac{1}{2} S_{55}, \quad S_{66}^{\mathrm{cp}}=\frac{1}{G_{x y}}=S_{55},
\end{gathered}
$$

where

$$
\begin{gathered}
a=\frac{S_{11}+S_{22}}{S_{11} S_{22}-\left(S_{12}\right)^{2}}, b=-\frac{2 S_{12}}{S_{11}+S_{22}}, c=-\frac{S_{12}\left(S_{22}-S_{12}-S_{23}\right)+S_{11} S_{23}}{S_{11} S_{22}-\left(S_{12}\right)^{2}}, \\
d=\frac{S_{11}\left(S_{22}\right)^{2}-2\left(S_{12}\right)^{2}\left(S_{22}-S_{23}\right)-S_{11}\left(S_{23}\right)^{2}}{S_{11} S_{22}-\left(S_{12}\right)^{2}} .
\end{gathered}
$$

Replacing elastic compliances $S_{i j}$ in eqns (7), (8) by complex compliances $S_{i j}^{*}(p), i j=11,22,12,23,55$, results in the exact complex compliances of a regular $\mathrm{CP}$ xFRP laminate, i.e.

$$
\left[S_{i j}^{\mathrm{cp}}(p)\right]^{*}=\left[S_{i j}^{\mathrm{cp}}(p)\right]_{\mathrm{e}}^{\prime}+\mathrm{i}\left[S_{i j}^{\mathrm{cp}}(p)\right]_{\mathrm{e}}^{\prime \prime} \quad, \quad i j=11,33,12,13,55,66
$$

\section{Standard and inverse $L V C$ equations of CP xFRP}

Following the considerations presented in [2], one can formulate standard LVC equations of the homogenized regular CP xFRP laminate, satisfying the assumptions adopted in Section 2, in the following form

$$
\boldsymbol{\varepsilon}(t)=\widetilde{\mathbf{S}}(t) \otimes \boldsymbol{\sigma}(t),
$$

where $\boldsymbol{\sigma}(t), \boldsymbol{\varepsilon}(t)$ are stress and strain vectors vs. time variable $t$, and $\widetilde{\mathbf{S}}(t)$ is a viscoelastic compliance matrix containing six independent viscoelastic compliances

$$
\widetilde{S}_{i j}^{\mathrm{cp}}(t)=S_{i j}^{\mathrm{cp}}\left\{1+\alpha_{i j} \int_{0}^{t}\left[\omega_{0} F_{0}\left(\vartheta ; \tau_{0}\right)+\omega_{1} F\left(\vartheta ; \tau_{1}\right)+\omega_{2} F\left(\vartheta ; \tau_{2}\right)\right] d \vartheta\right\}
$$

with $i j=11,33,12,13,55,66$ and 


$$
\begin{aligned}
& F_{0}\left(t ; \tau_{0}\right)=\frac{1}{\tau_{0}} \int_{0}^{\infty} \exp \left(-\frac{u t}{\tau_{0}}\right) u \Lambda(u) d u, \Lambda(u)=\frac{1}{\pi \sqrt{u}(1+u)}, \\
& F\left(t ; \tau_{i}\right)=\frac{1}{\tau_{i}} \exp \left(-\frac{t}{\tau_{i}}\right), \quad i=1,2 .
\end{aligned}
$$

The symbols used in eqns (10)-(12) denote: $\otimes-$ a convolution operator, $F_{0}\left(t ; \tau_{0}\right)$ - a fractional exponential function $\Phi_{0.5}\left(t ; \tau_{0}\right)[8], F\left(t ; \tau_{i}\right)$ - a normal exponential function $\Phi_{1}\left(t ; \tau_{i}\right)$ [8], $\omega_{0}, \omega_{1}, \omega_{2}$ - long-term creep coefficients describing the $\mathrm{HWKK} / \mathrm{H}$ rheological model of the matrix, $\tau_{0}, \tau_{1}, \tau_{2}$ - retardation times $\left(\tau_{0}<<\tau_{1}<<\tau_{2}\right)$ describing the HWKK/H rheological model of the matrix, $S_{i j}^{\mathrm{cp}}, \quad i j=11,33,12,13,55,66$ - elastic compliances of the CP xFRP laminate, $\alpha_{i j}, \quad i j=11,33,12,13,55,66$ - relative long-term creep coefficients (RLTC) dependent of micro- and meso-structure of the laminate as well as of the viscoelastic properties of the matrix.

An analytical method for calculating RLTC coefficients for a UD xFRP composite has been presented in [2]. The method adopts the exact and approximate complex compliances of the composite and the elastic-viscoelastic analogy principle. In this study, the method developed in [2] will be extended onto regular CP xFRP laminates.

Based on eqns (11), (12) the approximate (predicted) complex compliances of the laminate are described by the formulae

$$
\begin{aligned}
& {\left[S_{i j}^{\mathrm{cp}}(p)\right]^{*}=\left[S_{i j}^{\mathrm{cp}}(p)\right]^{\prime}+\mathrm{i}\left[S_{i j}^{\mathrm{cp}}(p)\right]^{\prime \prime}, \quad i j=11,33,12,13,55,66,} \\
& {\left[S_{i j}^{\mathrm{cp}}(p)\right]^{\prime}=S_{i j}^{\mathrm{cp}}\left\{1+\alpha_{i j}\left[\omega_{0} f_{0}\left(p \tau_{0}\right)+\omega_{1} f\left(p \tau_{1}\right)+\omega_{2} f\left(p \tau_{2}\right)\right]\right\},} \\
& {\left[S_{i j}^{\mathrm{cp}}(p)\right]^{\prime \prime}=-S_{i j}^{\mathrm{cp}} \alpha_{i j}\left[\omega_{0} g_{0}\left(p \tau_{0}\right)+\omega_{1} g\left(p \tau_{1}\right)+\omega_{2} g\left(p \tau_{2}\right)\right],}
\end{aligned}
$$

where [8]

$$
f_{0}(u)=\frac{\sqrt{2}+\sqrt{u}}{\sqrt{2}+2 \sqrt{u}+\sqrt{2} u}, g_{0}(u)=\frac{\sqrt{u}}{\sqrt{2}+2 \sqrt{u}+\sqrt{2} u}, f(u)=\frac{1}{1+u^{2}}, g(u)=\frac{u}{1+u^{2}} .
$$

The RLTC coefficients $\alpha_{i j}$ are derived from compatibility conditions put on the storage compliances at $p=0$. The final analytical formulae have the form [2]

$$
\alpha_{i j}=\frac{1}{\omega}\left\{\left[S_{i j}^{\mathrm{cp}}(0)\right]_{\mathrm{e}} / S_{i j}^{\mathrm{cp}}-1\right\}, \quad i j=11,33,12,13,55,66,
$$

where $\omega=\omega_{0}+\omega_{1}+\omega_{2}$ is the total long-term creep coefficient for the matrix.

The errors of fit of the predicted complex compliances to the exact complex compliances constitute the measure of modelling accuracy. The relative errors are defined by the formulae [2] 


$$
\begin{gathered}
\delta_{i j}^{\prime}=\frac{\sum_{k=1}^{n}\left|\left[S_{i j}^{\mathrm{cp}}\left(p_{k}\right)\right]^{\prime}-\left[S_{i j}^{\mathrm{cp}}\left(p_{k}\right)\right]_{\mathrm{e}}\right|}{\sum_{k=1}^{n}\left[S_{i j}^{\mathrm{cp}}\left(p_{k}\right)\right]_{\mathrm{e}}^{\prime}}, \quad \delta_{i j}^{\prime \prime}=\frac{\sum_{k=1}^{n}\left|\left[S_{i j}^{\mathrm{cp}}\left(p_{k}\right)\right] "-\left[S_{i j}^{\prime \prime}\left(p_{k}\right)\right]_{\mathrm{e}}^{\prime \prime}\right|}{\sum_{k=1}^{n}\left[S_{i j}^{\mathrm{cp}}\left(p_{k}\right)\right]_{\mathrm{e}}^{\prime}}, \\
i j=11,33,12,13,55,66,
\end{gathered}
$$

where $p_{k}, k=1,2, \ldots, n$, are the collocation points selected uniformly in the interval $p \in\left[p_{\min }, p_{\max }\right]$ in a logarithmic scale of frequency $p$.

Following the considerations presented in [2] and eqns (3), (4), inverse LVC equations of the homogenized regular CP xFRP laminate, satisfying the assumptions adopted in point 2 , are predicted in the following form

$$
\boldsymbol{\sigma}(t)=\widetilde{\mathbf{C}}(t) \otimes \boldsymbol{\varepsilon}(t)
$$

where $\tilde{\mathbf{C}}(t)$ is a viscoelastic stiffness matrix of structure analogous to $\widetilde{\mathbf{S}}(t)$, containing six independent viscoelastic moduli

$$
\begin{gathered}
\widetilde{C}_{i j}^{\mathrm{cp}}(t)=C_{i j}^{\mathrm{cp}}\left\{1-\beta_{i j} \int_{0}^{t}\left[\eta_{0} F_{0}\left(\vartheta ; \theta_{0}\right)+\eta_{1} F\left(\vartheta ; \theta_{1}\right)+\eta_{2} F\left(\vartheta ; \theta_{2}\right)\right] d \vartheta\right\}, \\
i j=11,33,12,13,55,66
\end{gathered}
$$

The symbols used in eqns (18) denote: $C_{i j}^{\mathrm{cp}}, \quad i j=11,33,12,13,55,66$ - elastic moduli defined by eqns (4), $\eta_{0}, \eta_{1}, \eta_{2} \quad$ - long-term relaxation coefficients describing the $\mathrm{HWKK} / \mathrm{H}$ rheological model for the matrix [3], $\theta_{0}, \theta_{1}, \theta_{2}-$ relaxation times $\left(\theta_{0}<<\theta_{1}<<\theta_{2}\right)$ describing the HWKK/H rheological model for the matrix [3], $\beta_{i j}, i j=11,33,12,13,55,66$ - relative long-term relaxation coefficients (RLTR) dependent of micro- and meso-structure of the laminate as well as of the viscoelastic properties of the matrix. An analytic algorithm for conversion of the standard viscoelasticity constants $\omega_{0}, \omega_{1}, \omega_{2}, \tau_{0}, \tau_{1}, \tau_{2}$ into the inverse viscoelasticity constants $\eta_{0}, \eta_{1}, \eta_{2}, \theta_{0}, \theta_{1}, \theta_{2}$ is presented in [2].

An analytical method for calculating RLTR coefficients for a UD xFRP composite has been developed in [3]. The method adopts the exact and approximate complex moduli of the composite and the relation between the complex compliance matrix $\mathbf{S}^{*}(p)$ and the complex stiffness matrix $\mathbf{C}^{*}(p)$. The relation between the complex compliance matrix and the complex stiffness matrix

$$
\mathbf{C}^{*}(p)=\left[\mathbf{S}^{*}(p)\right]^{-1}
$$

gives the exact complex moduli

$$
\left[C_{i j}^{\mathrm{cp}}(p)\right]^{*}=\left[C_{i j}^{\mathrm{cp}}(p)\right]_{\mathrm{e}}^{\prime}+\mathrm{i}\left[C_{i j}^{\mathrm{cp}}(p)\right]_{\mathrm{e}}^{\prime \prime} \quad, \quad i j=11,33,12,13,55,66
$$


The RLTR coefficients $\beta_{i j}, i j=11,33,12,13,55,66$, are derived by equating the approximate and exact storage moduli at frequency $p=0$. The final formulae have the form [3]

$$
\beta_{i j}=\frac{1}{\eta}\left\{1-\left[C_{i j}^{\mathbf{c p}}(0)\right]_{\mathrm{e}}^{\prime} / C_{i j}^{\mathbf{c p}}\right\}, \quad i j=11,3312,13,55,66
$$

where $\eta=\eta_{0}+\eta_{1}+\eta_{2}$ is the total long-term relaxation coefficient of the matrix.

The relative fit errors for the approximate and exact complex moduli are defined analogically to eqn (16).

\section{A numerical example}

A regular CP CFRP laminate of $[0 / 90]_{n \mathrm{~S}}, n \geq 4$ configuration, denoted with the symbol CP U/E53, is examined. Each ply is the UD U/E53 composite, considered previously in $[2,3]$. The matrix, made of Epidian 53 epoxide resin, is reinforced with UTS 5631 carbon fibres produced by Tenax Fibers. The elasticity and viscoelasticity constants of the matrix equal $[2,3]$

$$
\begin{gathered}
E=3.1 \mathrm{GPa}, v=0.42, \\
\omega_{0}=0.23, \omega_{1}=0.36, \omega_{2}=0.11, \tau_{0}=360^{\prime}, \tau_{1}=13000^{\prime}, \tau_{2}=310000^{\prime}, \\
\eta_{0}=0.19, \eta_{1}=0.19, \eta_{2}=0.04, \theta_{0}=240^{\prime}, \theta_{1}=9500^{\prime}, \theta_{2}=319500^{\prime} .
\end{gathered}
$$

The elasticity constants of a monotropic UTS 5631 carbon fibre equal $[2,3]$

$$
\bar{E}_{1}=234 \mathrm{GPa}, \bar{E}_{2}=6.6 \mathrm{GPa}, \bar{v}_{32}=0.36,{\overline{v_{21}}}=0.11, \bar{G}_{12}=10.6 \mathrm{GPa} .
$$

The real fibre volume fraction equals $f=0.50$.

The algorithms for calculation RLTC and RLTR coefficients have been programmed in Pascal. For the CP U/E53 laminate one obtains the effective elasticity constants and the RLTC and RLTR coefficients of the following values (given here with technical accuracy):

$$
\begin{gathered}
E_{x}=E_{y}=62.2 \mathrm{GPa}, z=7.3 \mathrm{GPa}, v_{z x}=v_{z y}=0.47, v_{y x}=v_{x y}=0.02, \\
G_{x z}=G_{y z}=2.1 \mathrm{GPa}, G_{x y}=2.6 \mathrm{GPa}, \\
\alpha_{11}=0.02, \alpha_{33}=0.12, \alpha_{12}=-0.20, \alpha_{13}=0.24, \alpha_{55}=0.61, \alpha_{66}=0.79, \\
\beta_{11}=0.02, \beta_{33}=0.16, \beta_{12}=-0.12, \beta_{13}=-0.17, \beta_{55}=0.72, \beta_{66}=0.86 .
\end{gathered}
$$

The exemplary diagram presenting the selected storage and loss compliance of the laminate vs. circular frequency $p$ in a semi-logarithmic scale is shown in Fig. 1. The fit errors do not exceed $0.42 \%$. Owing to very small fit errors, the diagrams reflect both the exact and predicted compliances.

The diagrams of the selected storage and loss modulus of the CP U/E53 laminate vs. circular frequency $p$ are shown in Fig. 2. The fit errors do not exceed $0.61 \%$. Owing to very small fit errors, the diagrams reflect both the exact and predicted moduli. 


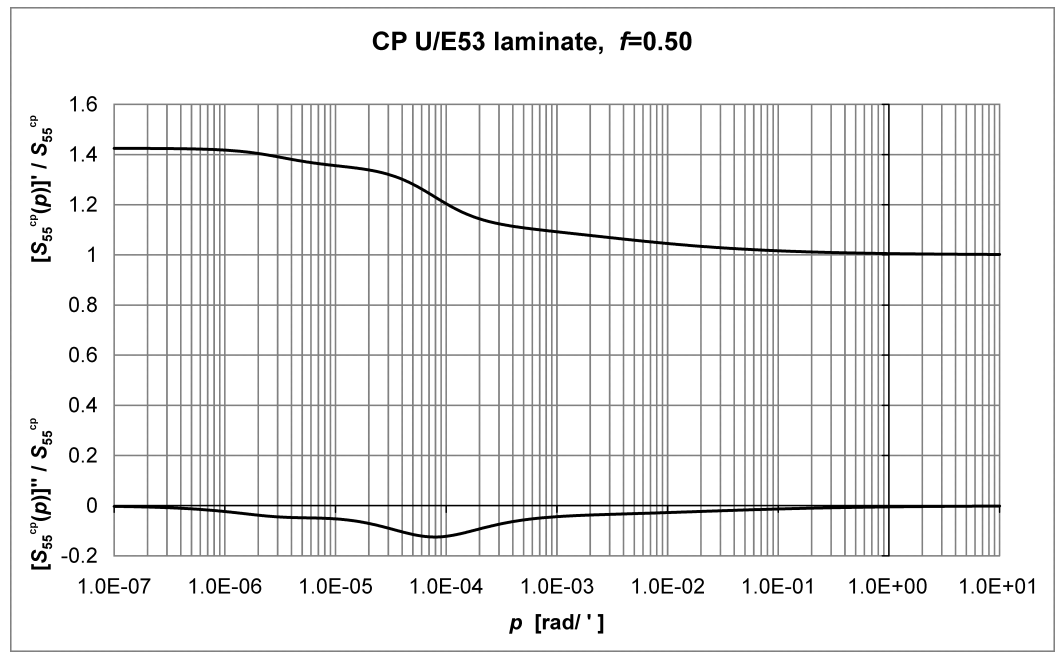

Figure 1: The relative storage and loss compliances of the CP U/E53 for $i j=55$.

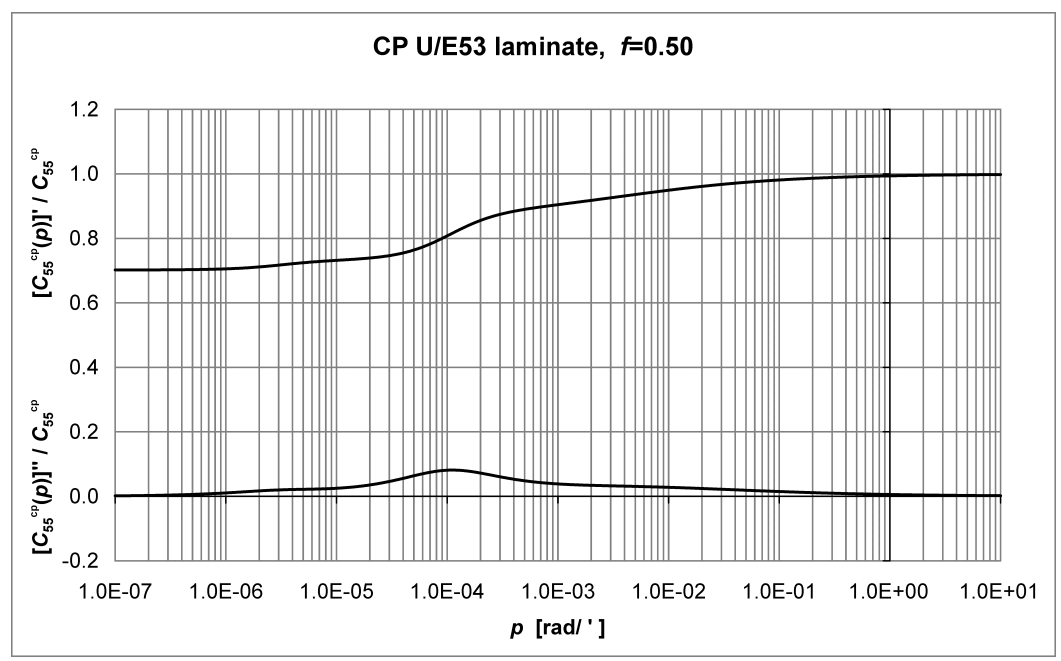

Figure 2: $\quad$ The relative storage and loss moduli of the CP U/E53 for $i j=55$.

\section{Final conclusions}

The study formulates a new approach to viscoelastic modelling of regular crossply polymer-matrix laminates. The matrix is a viscoelastic isotropic material described by the $\mathrm{HWKK} / \mathrm{H}$ rheological model, while the fibres are made of a monotropic elastic material. There are introduced the relative long-term creep coefficients and the relative long-term relaxation coefficients, mutually 
convertible. A number of viscoelasticity constants of regular cross-ply polymermatrix laminates has been reduced to minimum. The sets of RLTC and RLTR coefficients are calculated fully analytically via employing the exact stiffness theory of the laminate, the elastic--viscoelastic analogy principle and the relations between the viscoelastic compliance and viscoelastic stiffness matrices.

The calculation algorithms have been computerized and used to calculate the RLTC and RLTR coefficients for a number of CP xFRP laminates. The results for the selected laminated are presented in the study. Correctness, high accuracy and practical usability of the algorithms have been fully confirmed.

\section{Acknowledgement}

This work has been supported by the Ministry of Science and Higher Education, Poland, through grant No. R10 00101 . This support is gratefully acknowledged.

\section{References}

[1] Kiczko A., Klasztorny M., Ochelski St., A methodology of rheological experiments of polymer-matrix laminates to scientific purposes [in Polish], Scientific Books of Warsaw Univ. Techn., Series Mechanics, No. 219, pp. 103-114, 2007.

[2] Klasztorny M., Piekarski R., Witemberg-Perzyk D., Standard constitutive equations of linear viscoelasticity of UD xFRP composites, Scientific Books of Warsaw Univ. Techn., Series Mechanics, No. 219, pp. 275-288, 2007.

[3] Klasztorny M., Piekarski R., Inverse constitutive equations of linear viscoelasticity of UD xFRP composites, Scientific Books of Warsaw Univ. Techn., Series Mechanics, No. 219, pp. 289-304, 2007.

[4] Jones R. M., Mechanics of composite materials, Taylor \& Francis: London, 1999.

[5] Goracy K, Klasztorny M., Viscoelastic modelling of hardening plastics, Scientific Books of Warsaw Univ. Techn., Series Mechanics, No. 219, pp. 231-250, 2007.

[6] Klasztorny M., Piekarski R., Advances in the quasi-exact stiffness theory of UD xFRP composites, Scientific Books of Warsaw Univ. Techn., Series Mechanics, No. 219, pp. 251-262, 2007.

[7] Niezgoda T., Klasztorny M., Gieleta R., Homogenization of regular crossply polymer-matrix laminates, $4^{\text {th }}$ Int. Conf. on High Performance Structures and Materials, 13-15 May 2007, The Algarve, Portugal [in press].

[8] Klasztorny M., Characteristics of fractional and normal exponential functions and their basic applications to rheology, Scientific Books of Warsaw Univ. Techn., Series Mechanics, No. 219, pp. 263-274, 2007. 